\title{
Caracterização e avaliação do potencial agronômico e nutricional de etnovariedades de cubiu (Solanum sessiliflorum Dunal) da Amazônia
}

\author{
Danilo Fernandes da SILVA FILHO ${ }^{1}$; Lucia Kiyoko Ozaki YUYAMA ${ }^{1}$; Jaime Paiva Lopes AGUIAR ${ }^{1}$; Maslova \\ Carmo OLIVEIRA²; Lúcia Helena Pinheiro MARTINS ${ }^{2}$
}

\begin{abstract}
RESUMO
O cubiu (Solanum sessiliflorum Dunal) é um importante recurso genético para o povo da Amazônia, porque produz frutos tradicionalmente utilizados como alimento, medicamento e cosméticos. Por se tratar de um excelente produto para o agronegócio, aprofundar o conhecimento sobre a espécie, será uma contribuição de grande valia para a sociedade da região. Este trabalho teve o objetivo de caracterizar e avaliar o potencial de diferentes etnovariedades (ETNs) por meio de descritores agronômicos e minerais dos seus frutos. O Experimento foi conduzido na Estação Experimental de Hortaliças do INPA, em Manaus, em solo Podzólico vermelho-amarelo, álico, textura arenosa e de baixa fertilidade. Adotou-se um delineamento experimental de blocos ao acaso, com 28 tratamentos (as ETNs) em quatro repetições. Os seguintes descritores agronômicos e minerais dos frutos foram avaliados: forma dos frutos, número de frutos, comprimento do fruto, largura do fruto, peso médio do fruto, espessura da polpa, potássio, zinco, manganês, sódio, ferro, magnésio e cálcio. As análises de variância detectaram diferenças significativas entre as etnovariedades para todos os caracteres. As 28 ETNs puderam ser classificadas em nove formatos diferentes de frutos. As plantas produziram em média $4 \mathrm{a} 89$ frutos, variando em peso de $18,5 \mathrm{a} 301 \mathrm{~g}$. Entre os micro-elementos analisados a concentração de ferro variou de 97,3 a 352,7 mg em $100 \mathrm{~g}$ da polpa in natura. Destacou-se em conteúdo deste elemento, a ETN3, originária de Barcelos (AM). Entre os macro-elementos, o potássio (variando entre 54,6 a 563,5 mg em 100g da polpa in natura) apresentou maior concentração nas 28 ETNs avaliadas. AETN9, originária de Lábrea (AM), foi a que produziu os frutos mais ricos nesse elemento. De maneira geral, as ETNs estudadas apresentam variabilidade genética ampla para ser explorada em programa de melhoramento do cubiu.
\end{abstract}

\section{PALAVRAS-CHAVE}

Solanaceae, recurso genético, fruto, minerais, melhoramento genético.

\section{Characterization and evaluation of the agronomic and nutritional potential of etbnovarieties of cubiu (Solanum sessiliflorum Dunal) in Amazonia}

\begin{abstract}
Cubiu (Solanum sessiliflorum Dunal), is an important genetic resource for the Amazonian people, because its fruit is traditionally usede for food, medicine and cosmetics. As na excellent product for agribusiness, it is important to study the species to contribuite to regional development. The agronomic and nutritional potential of different cubiu ethnovarietes (ETNs) was characterized and evaluated., in an experiment at INPA's Horticulture Experimental Station, Manaus, Amazonas, Brazil, on a sandy textured, low fertility, alic, red-yellow Podzol, in an experimental design of randomized blocks, with 28 treatments (the ETNs) and four repetitions, the following fruit agronomic and chemical composition characteristics were evaluated: fruit number, shape, length, diameter, weight, pulp thickness, potassium, zinc, manganese, sodium, iron, magnesium and calcium. There were significant differences among the ETNs for all characteristics. The 28 ETNs were could be classified into nine different fruit shape. Fruit number/plant varied from 4 to 89, and mean fruit weight from 18.5 to 301 . Among the macroelents, potassium bad the highest concentration in all 28 ETNs (varying from 54.6 to $563.5 \mathrm{mg}$ in $100 \mathrm{~g}$ fresh pulp). The ETN from Lábrea (AM) produced the fruits with highest concentrations of this element. Among the microelements, iron varied from 97.3 to 352 mcg and the ETN from Barcelos (AM) had the highest concentration. In general, the ETNs presented ample genetic variability to be exploited in a cubiu improvement program.
\end{abstract}

\section{KEYWORDS}

Solanaceae, genetic resources, fruit, chemical composition, agrobusiness.

${ }^{1}$ Pesquisadores do Instituto Nacional de Pesquisas da Amazônia-INPA, C.P. 478, 69011-970, Manaus, AM, Brasil. Fone +5592 643 1864, e-mail: danilo@inpa.gov.br ${ }^{2}$ Bolsista CNPq/INPA. 


\section{ACTA \\ AMAZONICA}

CARACTERIZAÇÃO E AVALIAÇÃO DO POTENCIAL AGRONÔMICO E NUTRICIONAL DE ETNOVARIEDADES DE

CUBIU (Solanum sessiliflorum DUNAL) DA AMAZÔNIA

\section{INTRODUÇÃO}

O cubiu (Solanum sessiliflorum Dunal) é um dos recursos genéticos nativos da Amazônia, que foi completamente domesticado pelos povos indígenas da região antes da chegada dos europeus (Schultes, 1984). Nas últimas décadas este material genético tem gerado inúmeros estudos, sendo que os mais importantes, desenvolvidos na Amazônia brasileira e peruana constam os de Pahlen (1977), Salick (1987) e Silva Filho et al. (1993). Atualmente, o manual técnico publicado por Silva Filho (1998), é o trabalho mais completo encontrado na literatura internacional, no qual descrevem o cultivo, seu melhoramento, industrialização, com ênfase à conservação da espécie.

Sob o ponto de vista econômico, o cubiu tem se constituído uma importante matéria-prima para a agroindústria moderna, porque a planta é rústica, fácil de ser cultivada, muito produtiva dependendo do genótipo cultivado, podendo atingir 100 toneladas por hectare de frutos (Silva Filho et al., 1996) que podem ser utilizados de múltiplas formas (sucos, doces, geléias, compotas, molhos para carnes de um modo geral, cosméticos e medicamentos caseiros ou industrializados com ação hipocolesterolêmica e hipoglicêmica (Silva Filho, 2002). Somam-se ainda, a existência de alguns conhecimentos sobre suas caraterísticas químicas e tecnológicas que permitem sua industrialização em maior escala. Por ser anual e bem adaptado aos solos das várzeas da Amazônia, é possível produzir frutos com pouco ou nenhum insumo, permitindo sua comercialização por preços bem acessíveis (Silva Filho, 1998).

Por outro lado, não basta somente a preocupação de se conservar germoplasma. Há, também, a necessidade prioritária de estudos para caracterizar as variações morfológicas e a composição química, que possibilite o conhecimento mais aprofundado da biologia da espécie e da constituição genética de suas etnovariedades, para serem exploradas em prol da melhoria da qualidade de vida dos pequenos agricultores da Amazônia.

Os agricultores familiares são os detentores do material genético em suas roças e sítios. Contudo, lhes falta o conhecimento sobre as potencialidades que porventura possam estar presente em suas plantas e não foram ainda identificadas (Silva Filho et al., 2003). Portanto, a caracterização de populações, tanto fenotípica quanto genotípica são relevantes para se conhecer sua estrutura genética e avaliar aquelas características que estejam ligadas ao processo produtivo (Frankel, 1989). Este trabalho teve o objetivo de caracterizar e avaliar o potencial de 28 etnovariedades do cubiu por meio dos descritores morfológicos e minerais dos seus frutos.

\section{MATERIAL E MÉTODOS}

Utilizou-se 28 etnovariedades (conjunto de populações domesticadas, sempre cultivadas, definidas por uma combinação específica de recursos genéticos originais, distribuição geográfica restrita e história étnica associada) de cubiu, da coleção mantida pelo INPA em Manaus, AM, procedentes de diversas partes da Amazônia brasileira, peruana e colombiana, conforme localização geográfica apresentada na Figura 1.

O experimento de campo foi realizado na Estação Experimental de Hortaliças Alejo von der Pahlen (EEH) do Instituto Nacional de Pesquisas da Amazônia (INPA), localizada no Km 14 da Rodovia AM 010 em Manaus. O solo dessa área é do tipo Podzólico Vermelho-Amarelo álico, textura arenosa, de baixa fertilidade. O clima local é caracterizado como "Afi" no esquema de Köppen, registrando $2.450 \mathrm{~mm}$ de chuva, com uma estação seca no período de julho a setembro (EMBRAPA, 1982).

A semeadura foi feita no mês de março do ano de 2000, utilizando-se bandejas de isopor com solo previamente autoclavado a $120^{\circ} \mathrm{C}$, por duas horas, sendo posteriormente colocadas em casa de vegetação. Trinta dias após a semeadura, realizou-se a repicagem das plântulas para recipientes mais espaçosos, deixando-se somente uma planta por recipiente.

O solo da área experimental foi preparado no início do mês de abril/2000, constando de uma aração e uma gradagem. As covas foram abertas com as seguintes dimensões: $0,20 \mathrm{~m}$ de comprimento, por $0,20 \mathrm{~m}$ de largura e $0,20 \mathrm{~m}$ de profundidade, em um espaçamento de 1,0 m entre as covas e 1,5 m entre as fileiras.

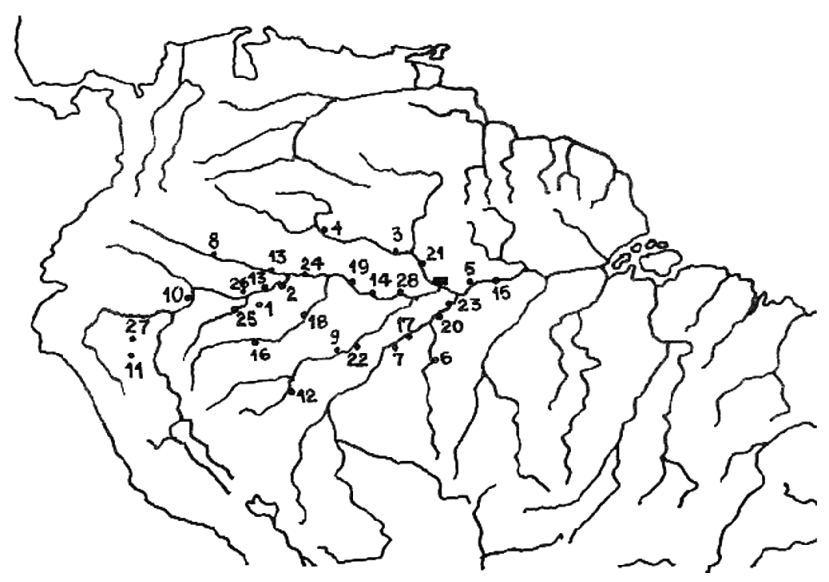

Figura 1 - Solanum sessiliflorum. Origem e localização geográfica de 28 etnovariedades de cubiu: Brasil: 1. Benjamin Constant/ AM; 2. São Paulo de Olivença/AM; 3. Barcelos/AM; 4. São Gabriel da Cachoeira/AM; 5. São Sebastião do Uatumã/AM; 6. Apui/AM; 7. Humaitá/AM; 9. Lábrea/AM; 12. Boca do Acre/AM; 13. Santo Antônio do Içá/AM; 14. Coari/AM; 15. Barreirinha/AM; 16. Eirunepé/AM; 17. Manicoré/AM; 18. Carauari/AM; 19. Tefé/AM; 20. Borba/AM; 21. Novo Airão/AM; 22. Canutama/AM; 23. Autazes/ AM; 24. Jutaí/AM; 25. Ataláia do Norte/AM e 28. Codajás/AM. Colômbia: 8. Putumayo e 26. Letícia; Peru: 10. Iquitos; 11. Tarapoto e 27. Yurimagua. 


\section{ACTA \\ AMAZONICA}

CARACTERIZAÇÃO E AVALIAÇÃO DO POTENCIAL

AGRONÔMICO E NUTRICIONAL DE ETNOVARIEDADES DE

CUBIU (Solanum sessiliflorum DUNAL) DA AMAZÔNIA
No ato do plantio foram aplicados em cada cova $2 \mathrm{~kg}$ de composto orgânico, $30 \mathrm{~g}$ de superfosfato triplo, $30 \mathrm{~g}$ de cloreto de potássio e $10 \mathrm{~g}$ de uréia. Aos 15 dias após o transplante fezse uma adubação em cobertura com $10 \mathrm{~g}$ de úreia por planta, repetindo-se essa prática até o momento em que as plantas iniciaram a produção de frutos.

O transplante foi realizado no início do mês de maio de 2000. Capinas, adubações complementares, irrigação, controle de doenças e pragas foram adotados sempre que necessários, de acordo com as recomendações de Silva Filho (1998).

Adotou-se o delineamento experimental em blocos casualizados com 28 tratamentos (etnovariedades) em quatro repetições. A unidade experimental foi constituída de uma área de $7,5 \mathrm{~m}^{2}$, contendo cinco plantas úteis.

A colheita dos frutos iniciou a partir do mês de novembro de 2000, estendendo-se até o mês de abril de 2001, quando apresentavam a coloração amarela (o ponto ideal de maturação para consumo in natura e em outros aproveitamentos na agroindústria). Nesse estádio de maturação, as sementes já estão fisiologicamente maduras e com o percentual de $100 \%$ de germinação (Silva Filho, 1994). Os frutos foram retirados dos ramos das plantas, cortando seus pedúnculos com tesoura de poda. Depois de retirados da planta, foram colocados em caixas plásticas com capacidade de $30 \mathrm{~kg}$, para evitar que fossem amassados.

As avaliações dos frutos para determinação dos caracteres agronômicos foram feitas nos laboratórios de Genética e Etnobiologia da Coordenação de Pesquisas em Ciências Agronômicas - CPCA. Utilizou-se técnicas de avaliação morfológicas baseadas nas evidências de caracteres constantes nos frutos das etnovariedades, adotando a terminologia citada em alguns trabalhos modernos de taxonomia, para estudos de espécies vegetais da família Solanaceae (Alcazar, 1981; Barroso et al., 1999; Radford, 1986; Stern, 1992). Os seguintes caracteres dos frutos foram observados nas 28 etnovariedades de cubiu: forma, número de frutos/planta (NF), comprimento (CF), largura (LF), espessura da polpa (EP) e peso médio dos frutos/ planta.

As análises químicas dos frutos do cubiu para determinação dos macro e microelementos minerais, foram realizadas no Laboratório de Nutrição e Físico-Química de Alimentos da Coordenação de Pesquisas de Ciências da Saúde, do Instituto Nacional de Pesquisas da Amazônia (INPA), em Manaus. Após o recebimento dos frutos devidamente identificados, os mesmos passaram por uma seleção, onde os frutos maduros isentos de cortes, furos e outros defeitos foram lavados por meio de imersão em água potável a temperatura ambiente $\left(27^{\circ} \mathrm{C}\right)$. Em seguida os frutos foram despolpados e tiveram a placenta (parte interna do fruto que contem as sementes e o suco da cavidade locular) e o epicarpo retirados com uma faca de aço inoxidável e triturados em um liquidificador elétrico, acondicionados em bandeja de aço inoxidável, congelados em freezer, a temperatura de $-20^{\circ} \mathrm{C}$ e liofilizados- operação de remoção da água por sublimação.

\section{Procedimentos para análise dos minerais nos frutos do cubiu:}

A fim de evitar a contaminação por metais que poderiam interferir na quantificação dos minerais, toda vidraria foi mantida em banho de ácido nítrico a $30 \%$ por no mínimo 12 horas, sendo em seguida enxaguada com água deionizada, por no mínimo seis vezes.

A determinação da umidade foi realizada por meio da liofilização, até o peso constante, seguindo as recomendações de Pitombo (1989).

O teor de minerais foi determinado pelo método de espectrometria de absorção atômica, por meio da leitura direta, em solução de amostras oxidadas a temperatura variando entre $150^{\circ} \mathrm{C} \mathrm{e} 200^{\circ} \mathrm{C}$, por via úmida, solubilizadas com ácido nítrico concentrado e perydrol 30 \% (Merck P.A.) e diluídas em água deionizada, segundo o método preconizado pelo Instituto Adolfo Lutz (IAL, 1985) e Manual PERKIN ELMER de 1985.

A leitura foi realizada diretamente nas soluções diluídas em espectrofotômetro de absorção atômica, PERKIN ELMER, modelo 1100B, com lâmpada de catodo oco, calibrado de acordo com as especificações do manual. O padrão de cada elemento mineral foi diluído (tritrisol Merck, em água desionizada e ácido nítrico $2 \%$ ) nas concentrações especificadas de acordo com o manual do equipamento.

As análises de variância dos caracteres número de frutos, largura do fruto, comprimento do fruto, espessura da polpa, peso médio dos frutos, potássio, zinco, manganês, sódio, ferro, magnésio e cálcio serviram para determinar as diferenças existentes entre as 28 etnovariedades, conforme o modelo matemático preconizado por Steel \& Torrie (1960). As análises de variâncias foram complementadas pelo teste de comparação múltipla de médias (teste de Tukey), em nível de $5 \%$ de probabilidade.

\section{RESULTADOS E DISCUSSÃO}

Os frutos das 28 ETN's apresentaram nove diferentes fenótipos (Figura 2). Segundo Barroso et al. (1999), são classificadas como baga ou solinídio. Cerca de $46,4 \%$ das etnovariedades produziram frutos de forma globosa, variando no tamanho desde pequeno a grande (Figura 2 e Tabela 1), com maior produção de frutos pequenos e médios. Em comprimento, os frutos variaram de 3,1 a 9,2 cm; na largura, 3,3 a 7,5 cm e, quanto a espessura da polpa, de 1,5 a $19,5 \mathrm{~mm}$. Os maiores frutos foram produzidos pelas ETN's 28, 12 e 3, originárias de Codajás, Estirão do Equador e de Barcelos, respectivamente.

As análises de variância detectaram diferenças significativas entre as etnovariedades para todas as características morfológicas e químicas estudadas nos frutos. Os coeficientes de variação estimados para essas características oscilaram na faixa de 1,12 \% a 20,5 \%. A predominância de valores tendendo 


\section{ACTA AMAZONICA}

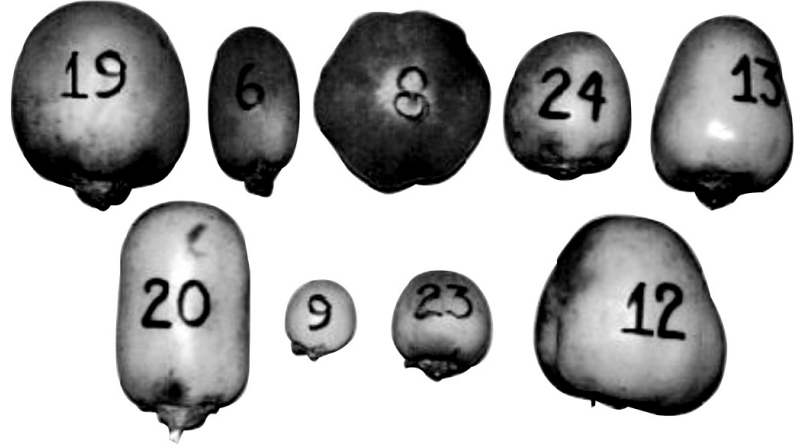

Figura 2 - Variação morfológica de frutos de cubiu (Solanum sessiliflorum Dunal) agrupando-se em nove fenótipos apresentados por 28 etnovariedades. para os menores percentuais (abaixo de $10 \%$ ) indica uma boa precisão experimental.

A produção significativa de frutos pequenos e médios observada nestas ETNs, pode estar relacionada com a preferência das populações tradicionais da Amazônia que os utilizam na preparação de sucos e/ou para substituir o limão, quando em falta. Por outro lado, o percentual menor de etnovariedades que produzem frutos de maior tamanho (acima de $200 \mathrm{~g}$ ), pode estar ligado aos processos de seleção praticados pelos índios e caboclos da Amazônia Ocidental em tempos mais recentes (Silva Filho, 1998).

Proporcionalmente, a espessura da polpa varia de acordo com o tamanho do fruto. As três caraterísticas dimensionais demonstram uma forte estabilidade fenotípica. Isto é importante para o programa de melhoramento do cubiu, porque é muito

Tabela 1 - Valores médios de caracteres morfológicos quantitativos avaliados em etnovariedades de cubiu (S. sessiliflorum Dunal). INPA/Manaus, 2002.

\begin{tabular}{llllll}
\hline \hline ETN & NM & CF $(\mathrm{cm})$ & LF $(\mathrm{cm})$ & PMF $(\mathrm{g})$ & EP $(\mathrm{mm})$ \\
\hline 01 & $4,0 \mathrm{o}$ & $6,1 \mathrm{i}$ & $5,7 \mathrm{ef}$ & $216,7 \mathrm{c}$ & $11,9 \mathrm{f}$ \\
02 & $17,7 \mathrm{efgh}$ & $6,2 \mathrm{i}$ & $5,5 \mathrm{fg}$ & $82,3 \mathrm{k}$ & $8,2 \mathrm{i}$ \\
03 & $5,7 \mathrm{no}$ & $5,7 \mathrm{kl}$ & $7,3 \mathrm{c}$ & $174,7 \mathrm{e}$ & $13,1 \mathrm{~d}$ \\
04 & $14,7 \mathrm{ghijk}$ & $7,4 \mathrm{f}$ & $4,4 \mathrm{k}$ & $85,7 \mathrm{j}$ & $5,1 \mathrm{~m}$ \\
05 & $7,0 \mathrm{mno}$ & $8,2 \mathrm{~d}$ & $5,6 \mathrm{efg}$ & $251,0 \mathrm{~b}$ & $12,9 \mathrm{de}$ \\
06 & $12,3 \mathrm{ijkl}$ & $8,1 \mathrm{~d}$ & $5,4 \mathrm{fgh}$ & $149, \mathrm{f}$ & $3,7 \mathrm{o}$ \\
07 & $10,7 \mathrm{klmn}$ & $9,1 \mathrm{~b}$ & $4,4 \mathrm{k}$ & $95,0 \mathrm{i}$ & $2,0 \mathrm{q}$ \\
08 & $16,0 \mathrm{fghij}$ & $5,8 \mathrm{jk}$ & $6,3 \mathrm{~d}$ & $150,0 \mathrm{f}$ & $7,1 \mathrm{j}$ \\
09 & $22,7 \mathrm{de}$ & $5,2 \mathrm{no}$ & $5,0 \mathrm{ij}$ & $55,0 \mathrm{p}$ & $5,7 \mathrm{l}$ \\
10 & $20,0 \mathrm{ef}$ & $5,3 \mathrm{mn}$ & $4,7 \mathrm{jk}$ & $64,7 \mathrm{o}$ & $2,7 \mathrm{p}$ \\
11 & $10,0 \mathrm{klmn}$ & $5,1 \mathrm{op}$ & $5,1 \mathrm{hi}$ & $75,0 \mathrm{~m}$ & $6,1 \mathrm{l}$ \\
12 & $8,0 \mathrm{lmno}$ & $8,4 \mathrm{c}$ & $8,7 \mathrm{~A}$ & $300,0 \mathrm{a}$ & $14,1 \mathrm{c}$ \\
13 & $12,7 \mathrm{hijkl}$ & $8,2 \mathrm{~d}$ & $5,5 \mathrm{fg}$ & $150,0 \mathrm{f}$ & $11,7 \mathrm{fg}$ \\
14 & $35,7 \mathrm{c}$ & $5,6 \mathrm{l}$ & $5,3 \mathrm{ghi}$ & $74,5 \mathrm{~m}$ & $4,7 \mathrm{mn}$ \\
15 & $20,0 \mathrm{ef}$ & $4,9 \mathrm{q}$ & $5,3 \mathrm{ghi}$ & $70,6 \mathrm{n}$ & $6,0 \mathrm{l}$ \\
16 & $35,7 \mathrm{c}$ & $5,3 \mathrm{mn}$ & $5,9 \mathrm{e}$ & $78,7 \mathrm{l}$ & $3,0 \mathrm{p}$ \\
17 & $13,0 \mathrm{hijkl}$ & $6,4 \mathrm{~h}$ & $6,3 \mathrm{~d}$ & $122,7 \mathrm{~g}$ & $11,3 \mathrm{~g}$ \\
18 & $17,3 \mathrm{fghi}$ & $5,0 \mathrm{pq}$ & $5,0 \mathrm{ij}$ & $76,0 \mathrm{~m}$ & $4,9 \mathrm{mn}$ \\
19 & $8,0 \mathrm{lmno}$ & $9,2 \mathrm{ab}$ & $7,7 \mathrm{~b}$ & $251,7 \mathrm{~b}$ & $1,5 \mathrm{r}$ \\
20 & $52,3 \mathrm{~b}$ & $3,1 \mathrm{r}$ & $3,4 \mathrm{l}$ & $18,5 \mathrm{q}$ & $1,8 \mathrm{qr}$ \\
21 & $14,7 \mathrm{ghijk}$ & $5,1 \mathrm{op}$ & $5,3 \mathrm{ghi}$ & $81,8 \mathrm{k}$ & $6,6 \mathrm{k}$ \\
22 & $18,0 \mathrm{efg}$ & $5,4 \mathrm{~m}$ & $5,4 \mathrm{fgh}$ & $76,7 \mathrm{~lm}$ & $4,5 \mathrm{n}$ \\
23 & $12,0 \mathrm{jklm}$ & $6,7 \mathrm{~g}$ & $7,7 \mathrm{a}$ & $94,5 \mathrm{i}$ & $16,5 \mathrm{~b}$ \\
24 & $25,7 \mathrm{~d}$ & $5,9 \mathrm{j}$ & $5,7 \mathrm{ef}$ & $97,7 \mathrm{~h}$ & $5,8 \mathrm{l}$ \\
25 & $15,0 \mathrm{ghijk}$ & $6,5 \mathrm{~h}$ & $6,4 \mathrm{~d}$ & $121,0 \mathrm{~g}$ & $9,6 \mathrm{~h}$ \\
26 & $89,3 \mathrm{a}$ & $3,2 \mathrm{r}$ & $3,3 \mathrm{l}$ & $20,2 \mathrm{q}$ & $0,8 \mathrm{~s}$ \\
27 & $11,3 \mathrm{jklm}$ & $7,6 \mathrm{e}$ & $7,5 \mathrm{bc}$ & $207,0 \mathrm{~d}$ & $12,5 \mathrm{e}$ \\
\hline \hline 18 & $9,0 \mathrm{lmno}$ & $9,3 \mathrm{a}$ & $7,6 \mathrm{bc}$ & $301,0 \mathrm{a}$ & $19,5 \mathrm{a}$ \\
\hline
\end{tabular}

$\mathrm{ETN}=$ Etnovariedades, $\mathrm{NF}=$ número de frutos, $\mathrm{CF}=$ comprimento do fruto, $\mathrm{LF}=$ largura do fruto, $\mathrm{PMF}=$ peso médio do fruto, $\mathrm{EP}=$ espessura da polpa. 


\section{ACTA \\ AMAZONICA}

CARACTERIZACCÃO E AVALIAÇÃO DO POTENCIAL

AGRONÔMICO E NUTRICIONAL DE ETNOVARIEDADES DE

CUBIU (Solanum sessiliflorum DUNAL) DA AMAZÔNIA fácil praticar a seleção de frutos para quaisquer finalidades agroindustriais, baseando-se no seu tamanho e forma.

O rendimento estimado com base no número médio de frutos (NMF) e no peso médio dos frutos/planta (PMF), discrimina claramente as etnovariedades de cubiu (Tabela 1). A magnitude de variação média de NMF foi de 4 a 89 frutos/ planta e para o PMF de 18,5 a $301 \mathrm{~g}$. Quando se comparam estes resultados com aqueles obtidos por Pahlen (1977) e Silva Filho et al. (1996), observa-se que sempre em testes iniciais, o desempenho em produti vidade em populações de cubiu (mesmo dentro do Estado do Amazonas), em ambiente diferente, é baixa. A partir do uso de genótipos descendentes das plantas introduzidas, as gerações futuras manifestam ganhos em produtividade de até $75 \%$, em número de frutos. O caráter PMF em cubiu sempre demonstra estabilidade fenotípica (Silva Filho et al., 1999).

Uma análise exclusiva para cada elemento mineral contido na polpa de cubiu (Tabela 2) demonstra uma ampla variação destes constituintes nutricionais entre as diferentes etnovariedades analisadas, constatada pelo desvio padrão (Dp).

Tabela 2 - Teores médios de macro e micro-elementos na composição química dos frutos das 28 etnovariedades de cubiu da Amazônia. Manaus/2002.

\begin{tabular}{|c|c|c|c|c|c|c|c|}
\hline ETN & $\mathrm{K}(\mathrm{mg})$ & $\mathrm{Zn}(\mathrm{g})$ & $\mathrm{Mn}(\mathrm{g})$ & $\mathrm{Na}(\mathrm{g})$ & $\mathrm{Fe}(\mathrm{g})$ & $M g(m g)$ & $\mathrm{Ca}(\mathrm{g})$ \\
\hline 1 & 164,4hij & 264,3efghi & $0,01 f$ & $0,02 k$ & $108,0 f g$ & $11,6 \mathrm{efg}$ & $5,9 f g h i$ \\
\hline 2 & $375,2 b$ & $306,3 e f g$ & $0,09 a$ & $0,40 \mathrm{bcd}$ & 268,3abcde & $17,9 c$ & $13,7 a b$ \\
\hline 3 & $54,6 \mathrm{k}$ & $105,0 \mathrm{lmn}$ & 0,02 cdef & $0,07 j k$ & $97,3 \mathrm{~g}$ & $4,3 \mathrm{~h}$ & $4,1 i$ \\
\hline 4 & 210,8 defgh & $327,3 \mathrm{cdef}$ & $0,01 f$ & $0,35 \mathrm{bcde}$ & 157,0 efg & 12,7 efg & 7,5efgi \\
\hline 5 & 187,2ghi & $154,3 \mathrm{klm}$ & 0,02 cdef & 0,16hijk & $196,7 c d e f g$ & $13,4 \mathrm{cdefg}$ & $6,4 \mathrm{fghi}$ \\
\hline 6 & $239,2 \mathrm{cdefg}$ & 313,0 defg & $0,05 b c$ & $0,07 \mathrm{jk}$ & $288,3 a b c d$ & $13,5 \mathrm{cdefg}$ & 8,6 efgh \\
\hline 7 & $189,9 \mathrm{fgh}$ & 235,3ghijk & $0,03 c d e f$ & 0,15hijk & $212,0 \mathrm{bcdef}$ & 12,9defg & 6,9efghi \\
\hline 8 & 253,9 cde & $427,0 \mathrm{~b}$ & $0,09 a$ & $0,19 \mathrm{ijk}$ & $165,7 e f g$ & $14,4 c d e f$ & $6,1 \mathrm{fghi}$ \\
\hline 9 & $563,5 a$ & $623,7 a$ & $0,03 \mathrm{cdef}$ & $0,41 b c$ & $352,7 a$ & $32,6 a$ & $13,1 \mathrm{abc}$ \\
\hline 10 & 237,4 defg & $124,0 \mathrm{~lm}$ & 0,05 cde & $0,41 b c$ & 200,3 cdefg & $14,5 c d e f$ & $5,8 \mathrm{ghi}$ \\
\hline 11 & $245,4 \mathrm{cdefg}$ & $403,7 b c$ & $0,03 c d e f$ & $0,27 \mathrm{bcdefgh}$ & $296,7 a b c$ & $17,9 c$ & 7,1efghi \\
\hline 12 & 147,9hij & $103,3 \mathrm{lmn}$ & $0,01 f$ & $0,29 \mathrm{bcdefgh}$ & $290,7 a b c$ & $10,1 \mathrm{fg}$ & 9,3 cdefgh \\
\hline 13 & $124,5 i j$ & $27,0 n$ & 0,02 cdef & 0,25defghi & $194,3 \mathrm{cdefg}$ & 10,4 efg & $6,1 \mathrm{fghi}$ \\
\hline 14 & $300,9 c$ & $437,3 b$ & $0,02 \mathrm{cdef}$ & $0,30 \mathrm{bcdefg}$ & 242,0abcde & $13,5 \mathrm{cdefg}$ & 7,5efghi \\
\hline 15 & $251,1 c$ def & $78,3 \mathrm{mn}$ & $0,04 \mathrm{cde}$ & $0,32 \mathrm{bcdef}$ & 175,0 defg & $15,1 \mathrm{cde}$ & 7,6efghi \\
\hline 16 & $267,3 \mathrm{~cd}$ & $245,3 f g h i j$ & 0,02 cdef & $0,43 b$ & $334,3 a$ & $17,7 c d$ & 10,5 bcde \\
\hline 17 & 204,0efgh & 230,7ghijk & $0,03 c d e f$ & $0,03 \mathrm{k}$ & 241,7abcde & $11,5 \mathrm{efg}$ & 9,7cdefg \\
\hline 18 & $261,7 \mathrm{cde}$ & $394,0 \mathrm{bcd}$ & $0,01 f$ & 0,24efghi & 255,3abcde & $12,1 \mathrm{efg}$ & 9,4 cdefg \\
\hline 19 & 227,4 defg & 177,7ijkl & 0,02 cdef & $0,04 j k$ & $204,3 \mathrm{bcdefg}$ & $14,4 \mathrm{cdef}$ & 8,9defgh \\
\hline 20 & $387,8 \mathrm{~b}$ & 216,0hijk & $0,08 a b$ & $0,61 a$ & 260,0abcde & $25,2 b$ & $17,0 a$ \\
\hline 21 & 237,3 defg & $174,3 \mathrm{jkl}$ & $0,01 f$ & $0,29 \mathrm{bcdefgh}$ & 209,0bcdefg & 11,0 efg & 8,5 efgh \\
\hline 22 & $265,7 \mathrm{cde}$ & $401,0 b c$ & $0,03 \mathrm{cdef}$ & 0,23efghi & $200,3 \mathrm{cdefg}$ & $10,6 \mathrm{efg}$ & $6,2 \mathrm{fghi}$ \\
\hline 23 & $384,8 \mathrm{~b}$ & 232,0ghijk & $0,05 \mathrm{bcde}$ & $0,36 \mathrm{bcde}$ & 220,0 bcdef & $18,3 \mathrm{c}$ & $9,8 \mathrm{bcdef}$ \\
\hline 24 & 237,6 defg & 286,3 efgh & $0,08 a b$ & 0,19fghij & $315,0 a b$ & $13,5 \mathrm{cdefg}$ & $12,6 \mathrm{bcd}$ \\
\hline 25 & 186,4ghi & $336,0 \mathrm{cde}$ & 0,02 cdef & 0,15ghijk & $187,3 \mathrm{cdefg}$ & $10,6 \mathrm{cfg}$ & $5,4 h i$ \\
\hline 26 & 205,6defgh & 211,3hijk & $0,01 f$ & $0,06 \mathrm{jk}$ & $161,7 \mathrm{cfg}$ & $11,4 \mathrm{cfg}$ & 6,8cfghi \\
\hline 27 & 187,2ghi & $178,0 \mathrm{ijkl}$ & 0,02 cdef & 0,16ghijk & $196,7 c d e f g$ & $13,4 \mathrm{cdefg}$ & $6,4 \mathrm{fghi}$ \\
\hline 28 & $113,9 j k$ & $111,7 \mathrm{lmn}$ & $0,02 \mathrm{cdef}$ & 0,27 cdefgh & $123,0 \mathrm{fg}$ & $8,9 \mathrm{gh}$ & $4,5 i$ \\
\hline Média & 239,7357 & 254,4321 & 0,032857 & 0,239643 & 219,7714 & 14,05 & 8,264286 \\
\hline Dp & 98,87413 & 133,3822 & 0,024774 & 0,143152 & 65,19213 & 5,24528 & 2,977224 \\
\hline DRIs & $2000 \mathrm{mg}^{* *}$ & $11 \mathrm{mg}$ & $2,3 \mathrm{mg}$ & $500 \mathrm{mg}^{* *}$ & $8 \mathrm{mg}$ & $420 \mathrm{mg}$ & $1000 \mathrm{mg}$ \\
\hline
\end{tabular}

Médias seguidas pelas mesmas letras não diferem entre si em nível de $5 \%$ de probabilidade pelo teste de Tukey.

*DRIs Quantidade diária recomendada ao estágio de vida de 31-50 anos do sexo masculino (NAS/IOM, 2001).

** RDA, 1989. 19-50 ANOS.

$\mathrm{ETN}=$ Etnovariedades, $\mathrm{K}=$ potássio, $\mathrm{Zn}=$ zinco, $\mathrm{Mn}=$ manganês, $\mathrm{Na}=$ sódio, $\mathrm{Fe}=$ ferro, $\mathrm{Mg}=$ magnésio, $\mathrm{Ca}=$ cálcio. 
Considerando a recomendação diária para um homem adulto no estágio de vida entre 31-50 anos, os diferentes elementos minerais presentes no cubiu não atendem o valor preconizado (Tabela 2). Contudo, as concentrações de potássio e magnésio, na etnovariedade de cubiu originária de Lábrea, representam $12 \%$ e $7 \%$ respectivamente desta necessidade se consumidos cerca de $100 \mathrm{~g}$ da polpa in natura (Tabela 2).

No geral, entre os minerais, potássio destacou-se em termos de concentração, nas 28 ETNs avaliadas. A amplitude de variação deste elemento foi de 54,6 a 563,5 mg nas ETN3 e ETN9, originárias de Barcelos e Lábrea (AM), respectivamente.

O potássio é um elemento largamente distribuído nos alimentos por ser um dos principais constituintes essenciais das células vegetais. Estes valores encontrados nas etnovariedades de cubiu são superiores aos analisados em tomate, pimentão, berinjela, batata e em outros frutos muito consumidos na Amazônia tais como: graviola, cupuaçu, araçá boi, pupunha, manga e abricó. Salienta-se que a concentração desses elementos minerais está ainda condicionada ao trato cultural (adubação) e tipo de solo (Yuyama et al., 1997).

Quanto à concentração de zinco em frutos de cubiu, as análises mostraram uma variação de 27,0 a $623,7 \mathrm{mg}$ nas etnovariedades ETN13 e ETN9 originárias de Santo Antônio do Içá e Lábrea (AM), respectivamente. Várias etnovariedades apresentaram teores desse elemento, superiores a $400 \mathrm{mg}$. Ao comparar o teor de zinco encontrado nos frutos de cubiu com outros registrados em análises de diversos alimentos de origem vegetal, o cubiu pode ser considerado uma boa fonte desse elemento. Pesquisadores que estudaram a dieta alimentar da população de Manaus (Shrimpton \& Giugliano, 1979, Yuyama et al., 1992) relataram que ozinco é um fator limitante. Portanto, o cubiu pode ser uma fonte disponível desse elemento que está ao alcance de qualquer família que desejar utilizá-lo. O cubiu pode ser facilmente cultivado ou comprado de alguns feirantes e micros empresários de Manaus que vendem frutos frescos e polpa congelada por preços bem acessíveis.

Entre os microelementos determinados nos frutos de cubiu, o manganês foi encontrado em menor quantidade, mas evidenciou a diversidade entre as ETNs. As etnovariedades ETN9, ETN8 e ETN20 foram as que apresentaram maiores concentrações de manganês $(0,09 \mathrm{mg}, 0,09 \mathrm{mg}$ e $0,08 \mathrm{mg})$, respectivamente. Dos elementos minerais analisados o manganês foi o que apresentou em menor concentração (Tabela 2). Entretanto, quando comparado com outras solanáceas como a berinjela (S. melongena $\mathrm{L}$.) os valores se aproximam (Philippi, 2001).

Dentre os minerais mais consumidos pelos animais, o sódio se destaca (Van Guren, 1979). Entretanto, o conteúdo desse elemento nestas ETNs é baixo. A ETN20, originária de Borba (AM), foi a que apresentou maior concentração deste elemento $(0,61 \mathrm{mg})$. Valores ínfimos é de fundamental importância aos pacientes com restrição de sódio, tais como os hipertensos, constituindo assim em mais uma alternativa alimentar aos amazônidas.

Entre os microelementos analisados nestas ETNs de cubiu, o ferro não deixa de ter a sua contribuição. A amplitude de variação desse elemento nos frutos das etnovariedades foi de 97,3 a 352,7 mg. Destacou-se como mais rica em ferro, a ETN3, originária de Barcelos (AM). Normalmente as dietas dos grupos populacionais, particularmente os biologicamente vulneráveis, apresentam limitação na ingestão de micronutrientes, dentre eles Fe e Zn (Yuyama et al., 2000, 1999, Yuyama \& Cozzolino, 1995). Dessa forma, o cubiu pode ser mais uma opção para compor a dieta da população amazonense.

O teor de magnésio variou nas etnovariedades estudadas de 4,3 a 32,6 mg e a etnovariedade ETN9 procedente de Lábrea (AM) foi a que se destacou com maior concentração. Estes valores encontrados nos frutos de cubiu são considerados baixos, quando se compara com outros vegetais que concentram teores acima de $1000 \mathrm{mg}$ (amêndoas, bananas, favas entre outras). Entretanto, em comparação com o tomate, pimentão, beringela e a batata inglesa, que são espécies mais consumidas dentro da família Solanácea, o cubiu é a fonte de alimento mais rico neste elemento.

O conteúdo de cálcio presente nos frutos de cubiu variou de 4,1 a 17,0 mg. AETN20, procedente de Borba foi considerada a fonte mais rica desse elemento nas 28 ETNs pesquisadas. Quando comparado com os teores de cálcio concentrados no tomate, pimentão, berinjela, batata inglesa (Philippi, 2001), e outras hortaliças e frutas da Amazônia, os frutos de cubiu apresentam valores muito bons. Entretanto, o cálcio contido nos alimentos de origem vegetal apresenta baixa biodisponibilidade decorrente dos fatores antagonistas da dieta (Tucker, 1993).

\section{CONCLUSÕES}

De maneira geral, as 28 ETNs estudadas apresentam variabilidade genética ampla tanto nas suas características agronômicas quanto na composição mineral de seus frutos para ser explorada em programa de melhoramento do cubiu.

O cubiu, não atende as necessidades nutricionais de minerais de um homem adulto. Entretanto, constitui-se em excelente opção para somar com os demais alimentos para compor a dieta dos amazônidas, particularmente para os indivíduos com restrição a sódio.

\section{BIBLIOGRAFIA CITADA}

Alcazar, J.E. 1981. Genetic resources of tomatoes and wild relatives. Longman, Roma: 121p.

AOAC. 1995. Association of Official Analytical Chemists/Official methods of analyses. 16 ed., Arlington. 1141p.

Barroso, G.M.; Amorim, M.P.; Peixoto, A. L.; Ichaso, C.L.F. 1999. Frutos e sementes. Morfologia aplicada à Sistemática de Dicotiledôneas. Ed. UFV. Viçosa. 443p.

Cozzolino, S.M.F. 1997. Biodisponibilidade de minerais. Rev. Nutr. PUCCAMP. 10(2):87-98.

EMBRAPA. 1982. Boletim agrometeorológico. Manaus. EMBRAPA/ UEPAE, $22 \mathrm{p}$. 


\section{ACTA \\ AMAZONICA}

CARACTERIZAÇÃO E AVALIAÇÃO DO POTENCIAL

AGRONÔMICO E NUTRICIONAL DE ETNOVARIEDADES DE

CUBIU (Solanum sessiliflorum DUNAL) DA AMAZÔNIA
Frankel, O.H. 1989. Principals and strategies of evaluation. In. Brown, A.H.D., Frankel. O.H., Marshall, D.R., Williams, J.T. The use of plant genetic resources. Cambridge Univ. Press, Cambridge, 245-260.

Instituto Adolfo Lutz. 1985. Normas Analíticas do Instituto Adolfo Lutz. Análise de água e alimento. 3 ed. São Paulo. v. 1. $533 \mathrm{p}$.

NAS/IOM. 2001. Dietary Reference Intakes for Vitamin A, Vitamin K, Arsenic, Boron, Chromium, copper, Iodine, Iron, Manganese, Molybdenum, Nickel, Silicon, Vanadium, and Zinc. (http/books. nap.edu/books 0309072794/html/ 351.html).p.351-398, .

Pahlen, A.V.D. 1977. Cubiu (Solanum topiro Humbl. \& Bonpl.), uma fruteira da Amazônia. Acta Amazonica, 7:301-107.

Philippi, S.T. Tabela de composição de alimentos:suporte para decisão nutricional. Brasília:ANVISA, FINATEC/NUT-UnB, 2001, 133p.

Pitombo, R. N. M. 1989. A liofilização como técnica de conservação de material de pesquisa. Ciência e Cultura, 41(5): 427-431.

Radford, A.E. 1986. Fundamentals of plant systematics. Harper \& Row, Publishers, Inc. New York. 498p.

Salick, J. 1987. Cocona (Solanum sessiliflorum) production and breeding potentials of the peach-tomato. In: Wickens, N.H; Day, P. New crops for food and industry. Ed. Chapman and Hall. Cambridge. p. 258-264.

Schultes, R.E. 1984. Amazonian cultigens and their northward migrations in pre-Colombian times. In: Pre-bistoric plant migration. Harvard University Press. Cambridge. p. 19-38.

Shrimptom, R.; Giugliano, R. 1979. Consumo de alimentos e alguns nutrientes em Manaus. Acta amazonica, 9(1):1741.

Silva Filho, D.F. 1994. Variabilidade genética em 29 populações de cubiu (Solanum topiro Humbl. \& Bonpl. Solanaceae) avaliada na Zona da Mata do estado de Pernambuco. UFRPE: Recife, PE. Dissertação de Mestrado. 80 p.

Silva Filho, D.F. 2002. Discriminação de etnovariedades de cubiu (Solanum sessiliflorum Dunal Solanaceae) da Amazônia, com base em suas características morfológicas e químicas. INPA/UFAM. Manaus, AM. Tese de Doutorado. 117 p.

Silva Filho, D.F. 1998. Cocona (Solanum sessiliflorum Dunal): Cultivo y utilizacion. Caracas, Venezuela: Secretaria ProTempore. Tratado de Cooperacion Amazonica. 114p.

Silva Filho, D.F.; Andrade, J.S.; Clement, C.R.; Machado, F.M.; Noda, H. 1999. Correlações fenotípicas, genéticas e ambientais entre descritores morfológicos e químicos em frutos de cubiu (Solanum sessiliflorum Dunal) da Amazônia. Acta Amazonica, 29(4):503-511.

Silva Filho, D.F.; Anunciação Filho, C.J.; Noda, H.; Reis, O.V. 1996. Variabilidade genética em populações naturais de cubiu da Amazônia. Horticultura Brasileira. 14(1):9-15.
Silva Filho, D.F.; Anunciação Filho, C.J.; Noda, H.; Reis, O.V. 1997. Seleção de caracteres correlacionados em cubiu (Solanum sessiliflorum Dunal) empregando a análise de trilha. Acta Amazonica, 27(4)229-240.

Silva Filho, D.F.; Noda, H.; Clement, C.R. 1993. Genetic variability of economic characters in 30 accessions of cubiu (Solanum sessiliflorum Dunal) evaluated in Central Amazonia. Revista Brasileira de Genética. 16(2):409-417.

Steel, R.G.D.; Torrie, J.H. 1960. Principles and procedures of statistics, Mc-Graw-Hill. New York. 481p.

Stern, W.T. 1992. Botanical Latin. History, Grammar, Syntax, Terminology and Vocabulary. Ed. Hafner Publ. Comp. New York. 223p.

Tucker, G. A. 1993. Introduction. In: Seymour, G.B.; Taylor, J.E.; Tucker, G. A. Biochemistry of fruit ripening. Chapman \& Hall, Cambridge. p.3-43.

Van Guren, J.P. 1979. The chemistry of texture in fruits and vegetables. Journal of Texture Studies, 10:1-23.

Yuyama, K.; Aguiar, J. P. L., Yuyama, L. K. O. 2002. Camu-camu: um fruto fantástico como fonte de vitamina C. Acta Amazonica, 32(1):169-174.

Yuyama, L. K. O.; Aguiar, J. P. L.; Macedo, S. H. M.; Alencar, F. H.. Nagahama, D.; Favaro, D. I. T.; Vasconcelos, M. B.; Cozzolino, S. M. F. 2000. Avaliação da alimentação de pré-escolares de Barcelos e Ajuricaba, Estado do Amazonas. Revista do Instituto Adolfo Lutz, 59 (1/2) 27-32.

Yuyama, L. K. O.; Aguiar, J. P. L.; Yonekura, L.; Nagahama, D. ; Alencar, F. H. 1999. Perfil Nutricional da dieta dos préescolares do município de Nhamundá- AM, Brasil. Acta Amazonica, 29 (4): 651-654.

Yuyama, L. K. O.; Cozzolino, S. M. F. 1995. Determinação dos teores de $\mathrm{Zn}, \mathrm{Fe}, \mathrm{Ca}, \mathrm{Se}, \mathrm{Cu}, \mathrm{K}, \mathrm{Mg}$, e Mn na dieta Regional de Manaus, AM. Revista do Instituto Adolfo Lutz, 55 (1): 45 50 .

Yuyama, L.K. O.; Cozzolino, S.M.F.; Rocha, Y.R. 1992. Composição química e percentual de adequação de dieta regional de Manaus, AM. Acta Amazonica, 22(4):587-93.

Yuyama, L.K.O.; Aguiar, J.P.; Macedo, S.H.M.; Gioia, T.; Silva Filho, D.F.1997. Composição centesimal de diversas populações de cubiu (Solanum sessiliflorum Dunal) da Estação Experimental do Instituto Nacional de Pesquisas da Amazônia, INPA. In: Anais do II Simpósio Latino Americano de Ciências de Alimentos. Campinas, S. P., Brasil.

\section{RECEBIDO EM 31/10/2003 ACEITO EM 07/11/2005}


